\title{
Carbono, peso e volume de necromassa lenhosa em área de plano de manejo florestal
}

\section{Carbon, weight and volume of wood necromass in a forest management plan area}

\author{
Mathaus Messias Coimbra Limeira ${ }^{1}$ (D) $\bullet$ Rone da Silva Barbosa ${ }^{1}$ (D) $\bullet$ Eduardo Ganassoli Neto ${ }^{2}$ (D) \\ - Maria Cristina Bueno Coelho ${ }^{3}$ (D) Maurilio Antônio Varavallo ${ }^{3}$ (D) $\bullet$ Juliana Barilli ${ }^{3}$ (D) \\ - Marcos Vinicius Cardoso Silva ${ }^{4}$ (D) $\bullet$ Mauro Luiz Erpen ${ }^{5}$ (D)
}

Recibido: 28/11/2019 Aceptado: 31/03/2020Ｐublicado: 28/07/2020

\begin{abstract}
The necromass found in ecosystems maintains the carbon content. Following this guideline, quantification becomes a valuable attribute for efficient forest management. The study aimed to quantify a fallen and standing woody necromass in the soil, weight and carbon stock in the area of the native management plan as a subsidy to it. The sampling adopted uses the fixed area method and was carried out with the installation of a circular plot with a radius of 10 meters $\left(314 \mathrm{~m}^{2}\right)$. Twenty-seven plots were systematically distributed, resulting in the measurement of 806 individuals, whose inclusion criteria were diameter $\geq 5 \mathrm{~cm}$ and tactile-visual format the degree of deterioration of dead wood. The average volume of necromass in the region was $14.70 \mathrm{~m}^{3} \mathrm{ha}^{-1}$ with $5.37 \mathrm{mg} \mathrm{ha}^{-1}$ of necromass. This value has a carbon stock of approximately $2.6 \mathrm{mg} \mathrm{ha-1}$. This research showed that necromass distribution in the area occurs regularly and unevenly distributed throughout the study area. The material presented has $78 \%$ of individuals with less than twenty centimeters in diameter and, for the standing material, $96.15 \%$ of individuals with less than forty centimeters. Necromass contributes to reduce $\mathrm{CO}_{2}$ concentration in the atmosphere and decrease the greenhouse effect. Contributing to the maintenance of organic matter and organic carbon helps in the recovery and cycling of nutrients and highlights the importance of carbon fixation.
\end{abstract}

Key words: Spatial distribution, Normality, Cerrado.

1. Graduação em Engenharia Florestal, Universidade Federal do Tocantins; Gurupi - Tocantins, Brasil. mathauslimeira2010@gmail.com, ronedasilva7@gmail.com

2. Mestre em Engenharia Florestal; Gurupi - Tocantins, Brasil; engf.edu@gmail.com

3. Professor (a), Universidade Federal do Tocantins; Gurupi - Tocantins, Brasil. mariacristina@uft.edu.br, jubarilli@uft.edu.br, mavaravallo@gmail.com

4. Programa de Pós-Graduação em Ciências Florestais e Ambientais, Universidade Federal do Tocantins; Gurupi - Tocantins, Brasil. markuz@uft.edu.br

5. Professor, Instituto Federal de Ciência e Tecnologia do Tocantins; Gurupi - Tocantins, Brasil. mauro.luiz.erpen@gmail.com 


\section{Resumo}

A necromassa encontrada nos ecossistemas realizam a manutenção do estoque de carbono. Seguindo esta orientação a quantificação torna-se um atributo valioso para um manejo florestal eficiente. O estudo teve como objetivos quantificar a necromassa lenhosa caída e suspensa sobre o solo, peso e estoque de carbono em área de plano de manejo de nativas como subsidio ao mesmo. A amostragem adotada utiliza o método da área fixa, e foi conduzida através da instalação de uma área circular com raio de 10 metros $\left(314 \mathrm{~m}^{2}\right)$. Foram medidas 27 parcelas distribuídas sistematicamente, obtendo a medição de 806 indivíduos totais, cujo critério de inclusão é ter diâmetro $\geq$ que $5 \mathrm{~cm}$ e de forma tátil-visual o grau de decomposição da madeira morta. A média do volume de necromassa na região foi de $14,70 \mathrm{~m}^{3} \mathrm{ha}^{-1}$ com 5,37 $\mathrm{mg} \mathrm{ha}^{-1}$ de massa de necromassa. Tal valor apresenta um estoque de carbono de aproximadamente $2,6 \mathrm{mg} \mathrm{ha}^{-1}$. A pesquisou mostrou que a distribuição da necromassa na área ocorre de forma regular. Os níveis de necromassa são desiguais nas parcelas. O material caído apresenta 78 \% dos indivíduos menores que 20 centímetros de diâmetro, e para o material em pé 96,15 \% possuem diâmetro menor que 40 centímetros. A necromassa contribui para a redução da concentração do $\mathrm{CO}_{2}$ na atmosfera e para a diminuição do efeito estufa. Contribuindo para a manutenção, tanto da matéria orgânica quanto do carbono orgânico, auxiliando na recomposição e na ciclagem de nutrientes e evidencia a importância fixação de carbono.

Palabras clave: Distribuição espacial, Normalidade, Cerrado.

\section{Introdução}

As formações florestais possuem a capacidade de armazenar carbono na matéria orgânica de origem vegetal. Devido ao aumento do efeito-estufa e do aquecimento global, é de suma importância o estudo e o conhecimento sobre tais vegetações, podendo vir a ser uma possível solução para os problemas mencionados anteriormente. A compreensão sobre o sequestro de carbono da atmosfera, pode ser obtida através de estudos onde o fluxo de carbono no ecossistema florestal, bem como o estudo sobre os estoques de carbono em seus diversos compartimentos são levados em consideração.

As florestas servem como depósitos de carbono, que se encontram na biomassa, e vão sendo liberados de forma lenta para a atmosfera através da decomposição da matéria orgânica [1], [2]. Entretanto, as repetidas mudanças sobre a forma de uso do solo como queimadas, aumento da agricultura, agropecuária e urbanização tem liberado para a atmosfera uma grande quantidade de carbono. Estudos que envolvem o funcionamento destes ecossistemas, quantificação de estoque e fluxo de carbono, são relevantes para compreendermos a dinâmica do $\mathrm{CO}_{2}$ dentro do ciclo global do carbono [3], [4], [5].

A maior parte do carbono presente nas florestas está presente na biomassa presente na parte viva da biomassa Supõe-se que as formações florestais possuam aproximadamente $40 \%$ do carbono armazenado na biomassa viva e biomassa morta (necromassa) [6], sendo que avaliações sinalizam que a necromassa pode representar valores compreendidos entre 20 a $40 \%$ do carbono total estocado em florestas tropicais, possuindo grande controle na ciclagem deste elemento no ecossistema [7]. Entretanto é importante destacar que quantificar o carbono presente na parte morta, pode apresentar resultados que significam uma quantidade significativa do estoque máximo de carbono nas florestal, devendo ser visto com sua devida importância.

A madeira morta em ecossistemas florestais pode ser avaliada por suas caraterísticas qualitativas como as dimensões, a origem (espécie ou grupo taxonômico) e o grau de decomposição [8] e por suas características quantitativas como volume, peso de necromassa e peso de carbono.

O termo necromassa trata-se de diferentes tamanhos e tipos de material lenhoso, que pode ser encontrado sobre o solo das florestas, incluindo toras, gravetos, fragmentos de madeira, galhos e raízes, possuindo como critério mínimo de coleta apresentar valor igual ou superior a $5 \mathrm{~cm}$ de diâmetro. De acordo com [9] apenas 30 países realizam inventários de necromassa, o que representa $13 \%$ do total de países do mundo, sendo que mais de $77 \%$ dos inventários de necromassa foram iniciados após o ano de 2000, nos quais são utilizados diferentes métodos de amostragem e, geralmente, com uma intensidade amostral pequena. No Brasil, o primeiro trabalho de quantificação de necromassa foi publicado em 2012, o que indica que estudos com essa finalidade são bastante recentes no país [10].

A necromassa encontrada nos ecossistemas realizam a manutenção do estoque de carbono. Seguindo esta orientação a quantificação torna-se um atributo valioso para um manejo florestal eficiente. O conhecimento sobre a quantidade e velocidade de produção dos estoques, é importante para auxiliar na compreensão do funcionamento e consequente fragilidade deste componente florestal [11]. Diante disto o trabalho teve como objetivos quantificar a necromassa lenhosa caída e em pé sobre o solo, peso e estoque de carbono 
localizado no sul do Tocantins como subsidio ao plano de manejo florestal.

\section{Materiais e métodos}

A coleta de dados foi realizada na área do Plano de Manejo Florestal Sustentável. Tal área pertence a empresa POIANI \& BIANCHI LTDA, com localidade na cidade de Dueré, localizada no sul do estado do Tocantins (1120'38" S, 49¹6'14" O). A área objeto de estudo é denominada da Unidade de Produção Anual 1 - UPA 1, dividida em 18 faixas de aproximadamente 24 hectares cada.

A região possui uma classificação climática de Thornthwaite como sendo é BlwA'a', qualificado como clima úmido com moderada deficiência hídrica no inverno apresentando média anual $1500 \mathrm{~mm}$.

A área possui inclinação em direção aos percursos de agua, havendo altitude próxima aos 200 metros. A formação florestal de estudo está inserida na Bacia Hidrográfica do Rio Formoso, pertencente a Região Hidrográfica do Araguaia-Tocantins. Os solos da área podem ser classificados como Gleissolos de textura variada com solos aluviais, este tipo de solo é dominante em terras de baixo relevo e inundações periódicas, fator que determina sua caracterização [12]. O Manual Técnico da Vegetação Brasileira [13] classifica a vegetação local como Floresta Estacional Semidecidual Aluvial.

\section{Determinação da necromassa}

A estimativa de massa lenhosa morta (necromassa) é estabelecida por uma relação entre volume e densidade. A densidade $(\mu)$ é elaborada através da mensuração de volume de campo e massa seca, de peças coletadas em campo, respeitando a classificação da necromassa, para que se tenham densidades específicas em cada categoria. Todas peças têm seus diâmetros e graus de decomposição anotados.

Para o estudo da necromassa foi estabelecido a área da UPA 1 e todas as variáveis de interesse (diâmetro (tendo como inclusão o diâmetro $\geq 5$ centímetros), comprimento ou outras variáveis) das peças de necromassa presentes na área foram mensuradas [14], [15]. Nesse método de mensuração, a forma e tamanho das parcelas são constantes e tem duas dimensões: comprimento e largura [16]. É um método mais dispendioso e delongado [17], porém alcança resultados mais precisos, que podem ser comparados com outras variáveis biométricas e ambientais [15].
A área da UPA 1 tem 440 ha distribuídos em 18 faixas de 24 ha aproximadamente cada. Foi utilizado o método sistemático de área fixa para determinar a necromassa da área (figura 1), que consiste em demarcar as parcelas de forma previa, onde cada parcela possui um raio de 10 metros $\left(314 \mathrm{~m}^{2}\right)$, coletando os valores dos materiais considerados necromassa. Foram medidas 27 parcelas num total de $8.478,00 \mathrm{~m}^{2}$.

A determinação do volume foi por cubagem rigorosa de Smalian (Equação 1). Caso o material lenhoso estivesse tanto dentro como fora da parcela, era medido somente sua parte presente dentro da parcela.

$$
V=\frac{A S 1+A S 2}{2} * L
$$

$V$, volume em $\mathrm{m}^{3} ; A S 1$ e AS2, áreas seccionais com ou sem casca em $\mathrm{m}^{2}$; $L$, comprimento em metros.

O material lenhoso em pé, foi calculado segundo a formula sugerida por [18] para florestal manejada (Equação 2).

$$
V n=0,000073 * D A P^{2,04364} * L^{0,8541797}
$$

$V n$, volume da necromassa; $D A P$, diâmetro à 1,3 metros; $L$, comprimento

Para o cálculo de necromassa em $\mathrm{mg} \mathrm{ha}^{-1}$ foi utilizado a densidade básica do material (d) em $\mathrm{mg} \mathrm{m}^{-3}$ multiplicado pelo volume (V) em m ha-1 $^{-1}$ Equação 3).

$$
N=\left(\frac{A S 1+A S 2}{2} * L\right) * D
$$

$N$, necromassa em Mg ha-1; AS1 e AS2, áreas seccionais com ou sem casca em $\mathrm{m}^{2} ; L$, comprimento em metros; $D$, densidade básica do material em $\mathrm{Mg} \mathrm{m}^{3}$.

Foi utilizada a densidade básica para necromassa determinada por [19], obtida de [20], para diâmetro inferior a $10 \mathrm{~cm}$ (Tabela 1). Para as matérias mortas com diâmetro maior que 10 centímetros, a densidade utilizada foi determinada devido ao seu grau de decomposição de acordo com a metodologia proposta por [21].

O carbono foi determinado segundo a metodologia de [22] (Equação 4)

$$
C=N * 0,439
$$

C, carbono $\mathrm{Mg} \mathrm{ha}^{-1} ; \mathrm{N}$, valor da necromassa em $\mathrm{Mg} \mathrm{ha}^{-1}$ 


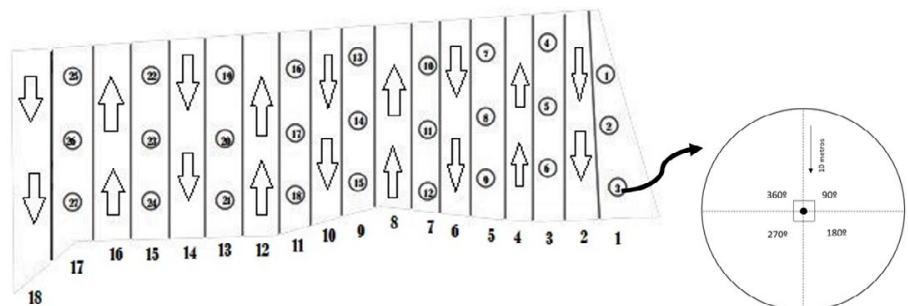

Figura 1. Desenho esquemático das parcelas dentro das faixas presente na UPA-1.

Figure 1. Schematic drawing of plots within strips present at UPA-1.

Foi determinada a distribuição da frequência de indivíduos, separada de acordo com a classe diametrica e subdividida pelo grau de decomposição. A quantidade de classes, foi obtida pela formula de Sturges (Equação 5).

$$
k=1+3,3 * \log N(5)
$$

k, número de classes; $N$, número total de individios

\section{Padrão espacial da distribuição da necromassa}

O padrão espacial da distribuição da necromassa foi analisado por meio do Índice de Morisita, usado para a determinação do padrão espacial de espécies [23] e adaptado por [22] para determinar o padrão espacial da distribuição das peças de necromassa e o padrão espacial do volume de necromassa, conforme apresentado na equação 6 .

$$
I_{\delta}=q\left(\frac{\sum_{i=1}^{q} X i^{2}-\sum_{i=1}^{q} X i}{\left(\sum_{i=1}^{q} X i\right)^{2}-\sum_{i=1}^{q} X i}\right)
$$

$I_{\delta}$ Ìndice de dispersão de morisita; $q$, número de unidades amostrais; $X i, V n$ por unidade de área $\left(\mathrm{m}^{3}\right.$ ha-1).

Tabela 1. Densidade de necromassa por classe de diâmetro e por classe de decomposição, de acordo com [20] e [21].

Table 1. Necromass density by diameter class and decomposition class, according to [20] e [21].

\begin{tabular}{|ccc|}
$\begin{array}{c}\text { Classe de diâmetro } \\
(\mathrm{cm})\end{array}$ & $\begin{array}{c}\text { Classe de } \\
\text { decomposição }\end{array}$ & $\begin{array}{c}\text { Densidade }(\mathrm{mg} \\
\left.\mathrm{m}^{-3}\right)\end{array}$ \\
\hline$<5$ & 1,2 e 3 & 0,36 \\
\hline 5 a 10 & 1,2 e 3 & 0,45 \\
\hline & 1 & 0,42 \\
$>10$ & 2 & 0,36 \\
& 3 & 0,30 \\
\hline
\end{tabular}

O valor de $F$ calculado (Equação 7) é comparado com $F$ tabelado e $N-1$ graus de liberdade no numerador e $\infty$ no denominador, onde $\mathrm{HO}$ a necromassa não se encontra distribuída de forma regular em toda a área e $\mathrm{H} 1$ : a necromassa está de forma regular em toda a área.

$$
F=\frac{I_{\delta}(n-1)+N-n}{N-1}
$$

\section{Determinação do nível de decomposição da Necromassa}

Através dos atributos exibidos decorrentes do nível de decomposição, os materiais: troncos, tocos, galhos, árvores mortas em pé ou caídas, foram classificadas de forma tátil visual, pelas características adotadas por [24], conforme Tabela 1.

\section{Teste de normalidade do estoque de Necromassa entre as parcelas}

O estoque de necromassa em volume para cada parcela foi determinado pelo teste de teste de Shapiro-Wilk ao nível de $5 \%$ de probabilidade de erro para testar a normalidade de volume de acordo com a Equação 8.

$$
W=\frac{b^{2}}{\sum_{i=1}^{n}(x i-\dot{x})^{2}}
$$

$x_{i}$, valores da amostra ordenados; $b$, constante.

Se $n$ é par:

$$
b=\sum_{i=1}^{\frac{n}{2}} a_{n-i+1} *\left(x_{(n-i+1)}-x i\right)
$$

Se $n$ é ímpar:

$$
b=\sum_{i=1}^{\frac{(n+1)}{2}} a_{n-i+1} *\left(x_{(n-i+1)}-x i \mid\right)
$$

Em que $a_{(n<+1)}$ valores tabelados.

\section{Análise dos dados}

Foram feitas as análises descritivas dos dados, agrupados de acordo com sua categoria necrovegetal, grau de decomposição, necromassa no chão e necromassa em pé e totais gerais, em cada parcela. Também a verificação da análise espacial do volume de necromassa e a normalidade da população amostrada 
Tabela 2. Classes de decomposição utilizadas para necromassa dos componentes arbóreos.

Table 2: Decomposition classes used for tree component necromass.

Classe de decomposição

D1

D2

D3
Características

Decomposição inicial - casca solta e/ou parcialmente ausente, alguns galhos presentes, grande parte coroa quebrado, fuste ainda firme.

Decomposição intermediária - alguns galhos presentes ou não, topo pode estar quebrado (> 2 m de altura residual), casca geralmente ausente e fuste ainda em pé, mas com alguns danos.

Decomposição avançada - galhos ausentes, casca ausente ou pouca casca, topo quebrado ( $\leq 2 \mathrm{~m}$ de altura residual) e fortemente deteriorado, fuste com cavidades extensas e ocas. via teste de Shapiro-Wilk, para nível de significância de $5 \%$. Foram utilizados os pacotes estatísticos Sigmaplot versão 12.0; minitab 19, Excel for Windows para análise e processamento dos dados.

\section{Resultados e discussão}

Para a frequência absoluta a necromassa caída (figura 2A) houve uma distribuição em forma de $U$ invertido nas duas primeiras classes diamétricas $5 \mathrm{~cm}$ a $12,5 \mathrm{~cm}$ e $12,5 \mathrm{~cm}$ a $20 \mathrm{~cm}$ para o material caído. Mostrando que há uma maior quantidade de necromassa de pequeno porte, encontrada no processo de decomposição intermediário. No material caído, as duas classes iniciais apresentam uma maior frequência de árvores medidas, onde a primeira classe apresenta $78 \%$ da amostragem de todo material caído, a segunda classe apresenta 15,4 $\%$, enquanto todas as demais classes juntas apresentam somente $6,6 \%$. A $9^{a}$ classe diamétrica do material caído não apresentou nenhum indivíduo, enquanto para o material $\mathrm{m}$ pé, a $4^{\mathrm{a}}$ e a $6^{\mathrm{a}}$ classe diamétrica, não apresentaram indivíduos.

Para necromassa caída, as duas classes diamétricas iniciais apresentam um maior valor volumétrico (figura $2 \mathrm{C})$, representando $45,2 \%\left(19,6 \mathrm{~m}^{3}\right)$ do volume total de estoque de necromassa, com destaque para o grau de decomposição $1 \mathrm{com} 49,2 \%\left(21,3 \mathrm{~m}^{3}\right)$. Já para a para a necromassa em pé (figura 2D), 52,5\% (17,6 $\left.\mathrm{m}^{3}\right)$ do volume total de necromassa está nas três primeiras classes com destaque para grau de decomposição 1 com $74,6 \%\left(25,1 \mathrm{~m}^{3}\right)$ do volume indicando que o estoque de necromassa existente na área é composto basicamente por material fino.

As demais classes diamétricas, juntamente com a amostragem da necromassa em pé (figura 2B), apresentaram uma distribuição exponencial negativa ("J" invertido), corroborando com a distribuição de diâmetros em estudos semelhantes a este em outras regiões no brasil e no mundo. [10] e [24] estudando a distribuição de frequência de diâmetros em regiões de Floresta Ombrófila Mista no estado do Paraná e em terras localizadas em Injune, Kiola e Warra na Austrália, respectivamente, encontraram distribuição de frequência semelhantes à deste trabalho.

O volume por faixas também foi classificado de acordo com o grau de decomposição da necromassa, e subclassificado em matéria caído ou em pé (Tabela 2) apresentou um valor médio de $18 \mathrm{~m}^{3}$ ha-1 $\pm 21,7$,tendo para ambos os casos o grau de decomposição 1 como proedominante $(51 \%$ necromassa caída e 69,1 \% para em pé), valor maior ao encontrado por [25] o qual registrou todos os galhos e troncos de madeira ou outro material lenhoso encontrado na área, caracterizada como parcela de coleta, com diâmetro igual ou superior a $2,5 \mathrm{~cm}$. Obtendo valores na região fitoecológica de Florestal Estacional Decidual de $14,9 \mathrm{~m}^{3} \mathrm{ha}^{-1}$, e inferior ao encontrado na Floresta Estacional Semidecidual, com valor de $30,5 \mathrm{~m}^{3} \mathrm{ha}^{-1}$, na Floresta Ombrófila Densa foi de $16,8 \mathrm{~m}^{3}$ ha- e na Restinga de $7,4 \mathrm{~m}^{3} \mathrm{ha}^{-1}$. Os valores encontrados em outras publicações sugerem médias de volume de 15,9 a 23,7 $\mathrm{m}^{3}$. ha-1 em Floresta Estacional Decidual no estado de Santa Catarina, ou 22,1 a 29,5 $\mathrm{m}^{3}$ ha $^{-1}$ em Floresta Ombrófila Densa no estado de SC e do AM, respectivamente. Os valores para necromassa foram extrapolados para metros cúbicos por hectare em cada faixa (Tabela 3 ).

O maior valor foi encontrado para necromassa em pé, em estágio inicial de decomposição, onde apresentou como maior valor $118,8 \mathrm{~m}^{3}$ ha-1 e um total de $266,9 \mathrm{~m}^{3} \mathrm{ha}^{-1}$. A necromassa em pé, dificilmente é encontrada no grau de decomposição 3 , devido a madeira não possuir mais sua estrutura de resistência, como forma de comprovar isso, foi somente encontrando o valor de $0,4 \mathrm{~m}^{3}$.ha-1 na faixa 4 . As demais faixas não apresentaram material lenhoso em pé, no grau de decomposição avançado (D3). O estoque de necromassa observado está, em sua maioria, em estágio inicial de decomposição, fazendo com que, independente da proporção, estas florestas tendem 


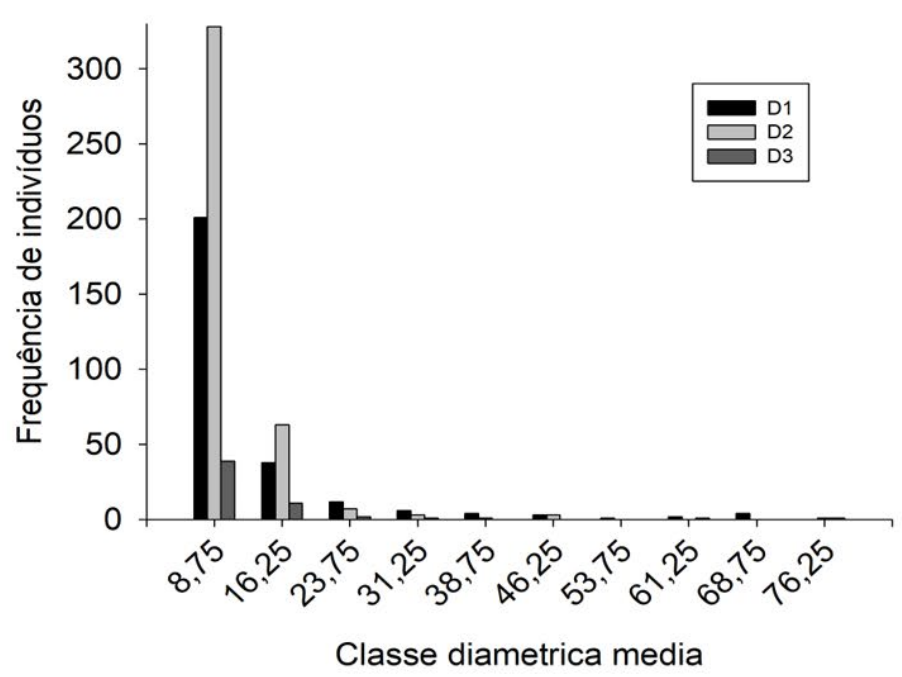

A

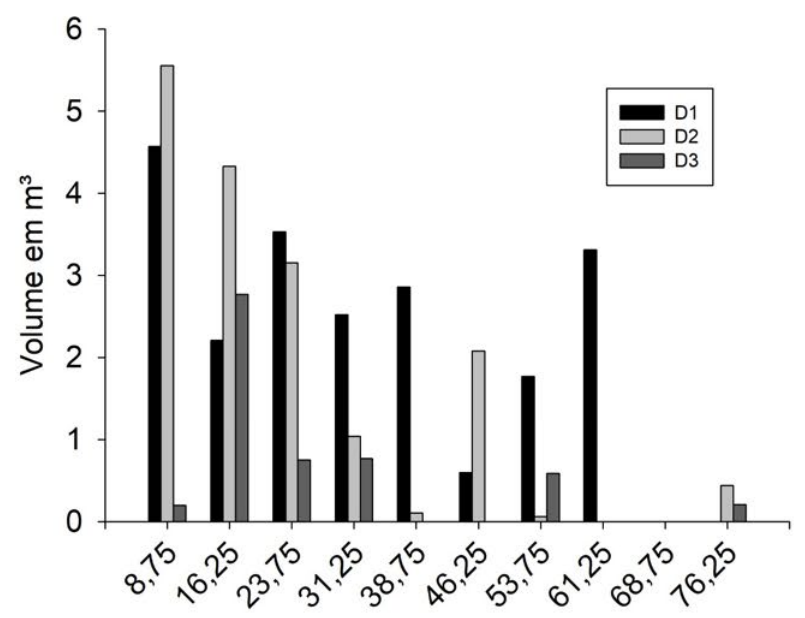

Centro de classe diametrica

C
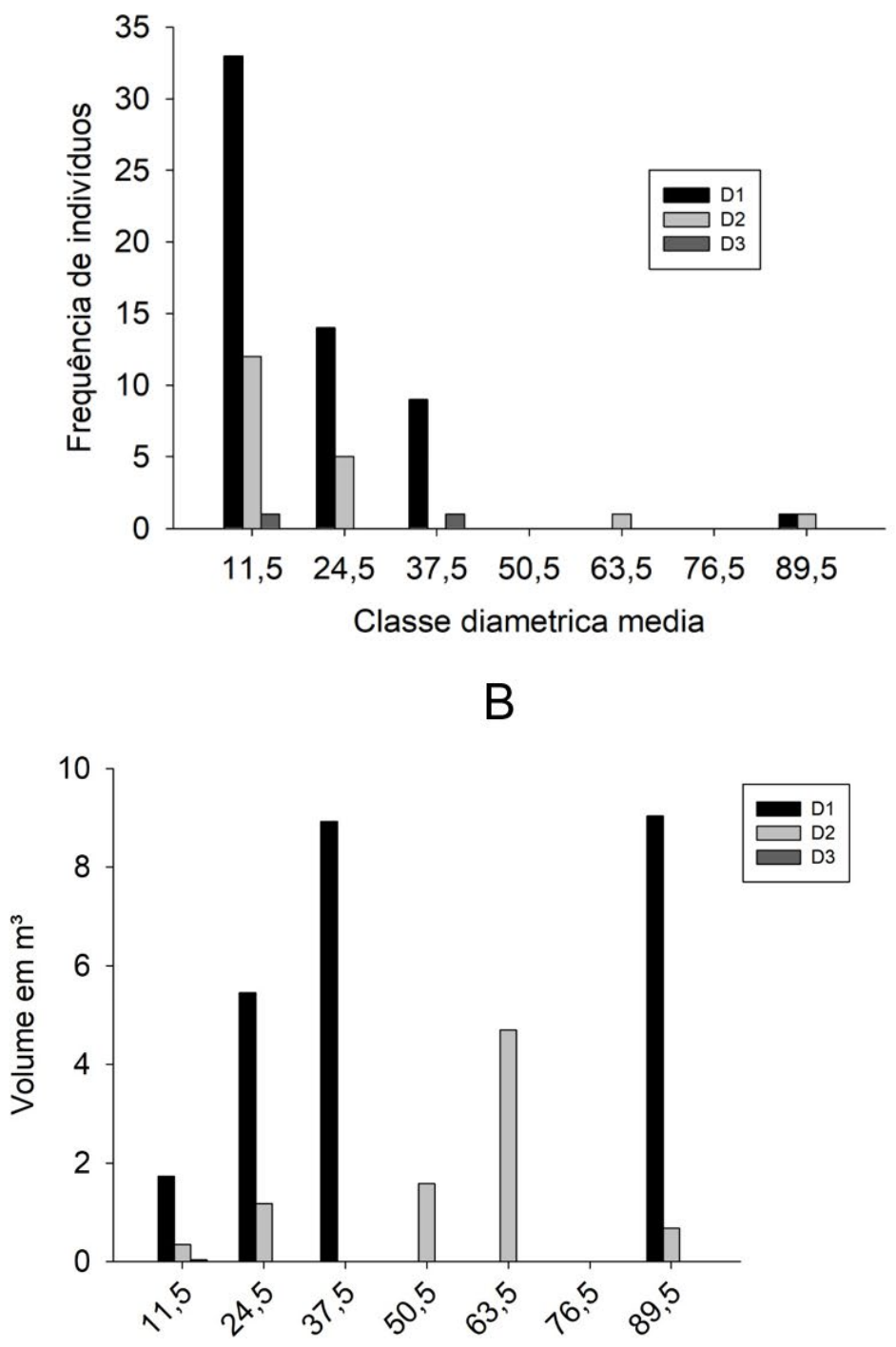

Centro de classe diametrica

Figura 2. Necromassa caida (A e C) e necromassa em pé (B e D) por grau de decomposiçāo (A e B) e em volume por classe diamètrica (C e D).

Figure 2. Fallen necromass ( $A$ and $C$ ) and standing necromass ( $B$ and $D)$ by degree of decomposition $(A$ and $B)$ and in volume by diameter class $(C$ and D).

mais à retenção de carbono a partir da decomposição da necromassa que à sua liberação.

O peso da necromassa foi obtido em mg.ha- ${ }^{-1}$, onde o somatório das vinte e sete amostras apresentaram um valor de 27,4 mg (sendo 16,4 mg deitado e 10,9 em pé). Ao extrapolar para hectare e realizar a média do peso, o valor apresentado é de $16,1 \mathrm{mg} \mathrm{ha}^{-1} \pm 18,4$. Valor semelhante ao encontrado por [26] com peso de 16,6 $\mathrm{mg} \mathrm{ha}^{-1}$ no cerrado do DF e valor semelhante aos obtidos por [27] que classificou as peças em "P" (diâmetros no intervalo de $2,0 \mathrm{~cm} \geq \mathrm{di} \geq 5,9 \mathrm{~cm})$, "M" $(6,0 \mathrm{~cm}>\mathrm{di} \geq$ $9,9 \mathrm{~cm}$ ) e as "G" (di $\geq 10,0 \mathrm{~cm}$ ) em cerrado sensu stricto de 4,89 $\mathrm{mg} \mathrm{ha}^{-1}$, enquanto em Floresta ombrófila densa montana, 2,8 $\mathrm{mg} \mathrm{ha}^{-1}( \pm 0,2)$ não manejada.
De acordo com [28], foram encontradas médias de necromassa acumulada de 3,2 a 6,6 mg.ha- ${ }^{-1}$ em Floresta Ombrófila Densa, ou 5,6 a 8,6 mg.ha-1 em Floresta Estacional Semidecidual ambas não manejada. [22] analisando o estoque de necromassa (com diâmetro de inclusão $\geq 10 \mathrm{~cm}$ ), encontrou o valor de $3,5 \pm 0,9 \mathrm{mg}$ ha 1, em região de Floresta Ombrófila Mista no Estado do Paraná em área sem manejo. Em Niquelândia -GO em área de Floresta Estacional Decidual sem exploração foi encontrado valor de necromassa em área não manejada variando de 1,0472 $\mathrm{mg} \mathrm{ha}^{-1} \mathrm{a} \pm 0,3403$ [29].

Já para o valor do carbono da necromassa foi obtido em $\mathrm{mg} \mathrm{ha}^{-1}$, onde o somatório das vinte e sete amostras apresentaram um valor de $14,1 \mathrm{mg}$ (sendo $8,5 \mathrm{mg}$ deitado 
Tabela 3. Volume da necromassa por faixa, por grau de decomposição e por categoria

Table 3: Necromass volume by strip, degree of decomposition and by category

\begin{tabular}{|c|c|c|c|c|c|c|}
\hline \multirow{2}{*}{$\begin{array}{l}\text { Faixas/Grau de } \\
\text { decomposição }\end{array}$} & \multicolumn{3}{|c|}{ Necromassa caída $m^{3}$ ha $^{-1}$} & \multicolumn{3}{|c|}{$\begin{array}{c}\text { Necromassa em pé } \\
\mathrm{m}^{3} \mathrm{ha}^{-1}\end{array}$} \\
\hline & D1 & D2 & D3 & D1 & D2 & D3 \\
\hline 1 & 30,45 & 23,86 & 2,71 & 13,50 & 0,65 & \multirow{9}{*}{0,41} \\
\hline 2 & 13,33 & 46,70 & 6,61 & 23,75 & 0,58 & \\
\hline 3 & 21,79 & 12,12 & 1,56 & 21,29 & 0,82 & \\
\hline 4 & 6,06 & 13,85 & 0,19 & 118,77 & 8,38 & \\
\hline 5 & 10,73 & 15,49 & 6,25 & 14,14 & 1,95 & \\
\hline 6 & 13,62 & 5,22 & 0,54 & 13,02 & - & \\
\hline 7 & 66,33 & 29,82 & 2,07 & 52,28 & 27,83 & \\
\hline 8 & 22,58 & 14,17 & 10,23 & 9,95 & 49,84 & \\
\hline 9 & 37,99 & 15,38 & 6,56 & 0,20 & - & \\
\hline Total & 222,88 & 176,61 & 36,72 & 266,9 & 90,05 & 0.41 \\
\hline Média & 24,764 & 19,62 & 4,08 & 29,656 & 12,864 & 0.41 \\
\hline
\end{tabular}

e 5,6 em pé). Ao extrapolar para hectare e realizar a média do peso, o valor apresentado é de $7,9 \mathrm{mg} \mathrm{ha}^{-1} \pm$ 4,3 . Os valores obtidos neste estudo para o estoque de carbono foram menores que os apresentados por [19] em estudo semelhante no Inventário Florístico Florestal de Santa Catarina para as mesmas regiões estudadas, como por exemplo 8,2 $\mathrm{mg} \mathrm{ha}^{-1}$ para a Floresta Estacional Decidual, 9,3 $\mathrm{mg} \mathrm{ha}^{-1}$ para a Floresta Ombrófila Densa e $3,3 \mathrm{mg} \mathrm{ha}^{-1}$ para a Restinga. [22] para a Floresta Ombrófila Mista encontrou $11,4 \mathrm{mg} \mathrm{ha}^{-1}$. [30] obtiveram um estoque de 8,9 mg.ha- ${ }^{1}$ em Floresta Estacional Semidecidual não manejada.

Quando comparado com áreas não exploradas na Amazônia Central considerando árvores mortas em pé e troncos caídos com diâmetro mínimo de $10 \mathrm{~cm}$, [31] encontraram valores superiores a este estudo, sendo que o volume médio encontrado varia de 44,4 a $\pm 16,2$ $\mathrm{m}^{3} \mathrm{ha}^{-1}$ com peso de 40,9 a $\pm 11,2 \mathrm{mg} \mathrm{ha}^{-1}$ e carbono de $19,6 \mathrm{a} \pm 5,4 \mathrm{mg}^{3} \mathrm{ha}^{-1}$.

Segundo os autores [32], a densidade da madeira dos resíduos lenhosos é um componente crucial na avaliação do acumulo de carbono, sendo necessário a realização de mais estimativas regionais. O nível de carbono utilizado neste estudo segue o valor sugerido e obtido por [22].

\section{Padrão Espacial da Distribuição da Necromassa}

O cálculo do padrão espacial das peças pelo Índice de Morisita resultou no valor 1,75 indicando não haver dependência espacial, pois nos cálculos considerou-se a alta concentração de unidades amostrais com valores de necromassa entre 0 e $30 \mathrm{~m}^{3}$ ha-1. Já para o valor de $\mathrm{F}$
(Ftab $=1,59$ e Fcal $=24,39)$ foi determinado um padrão agregado, no qual, a necromassa se encontra dispersa em toda a área.

Adicionado a essa análise, considerou-se também a análise visual da distribuição de necromassa. A partir da análise espacial do volume de necromassa, que compõe a Figura 4, em que se observam os padrões pontuais de necromassa em todas as unidades amostrais de estudo. Enquanto os pontos maiores simbolizam as unidades amostrais com maiores volumes de necromassa, os pontos de menor tamanho indicam as unidades amostrais de volumes inferiores.

É possível visualizar que a distribuição volumétrica da necromassa se encontram dispersa por toda a extensão da área de estudo estando arranjada em um padrão de forma agregada, indicando as maiores concentrações de volumétricas de necromassa nos extremos das faixas. Este resultado deve-se à complexidade e inconstância da variável de estudo. [33] ao mapear de modo contínuo e extenso a distribuição espacial da necromassa no Gatineau Park (cerca de 20 a $50 \mathrm{~km}$ a noroeste da cidade de Ottawa, no Canadá) observaram a mesma tendência, ou seja, esta variável distribuía-se de maneira desigual em toda a área de estudo.

Por meio do teste de Shapiro-Wilk, foi constatada a normalidade da variável volume $\left(\mathrm{m}^{3} \mathrm{ha}^{-1}\right)$ na área amostrada (composta pelas 27 parcelas). Pela regra de decisão do teste, Wcalculado $=0,811<W(0,05 ; 27)=0,923$, Assim, podemos afirmar com nível de significância de 5 $\%$ que a amostra provém de uma população irregular. 
Peso por grau de decomposição

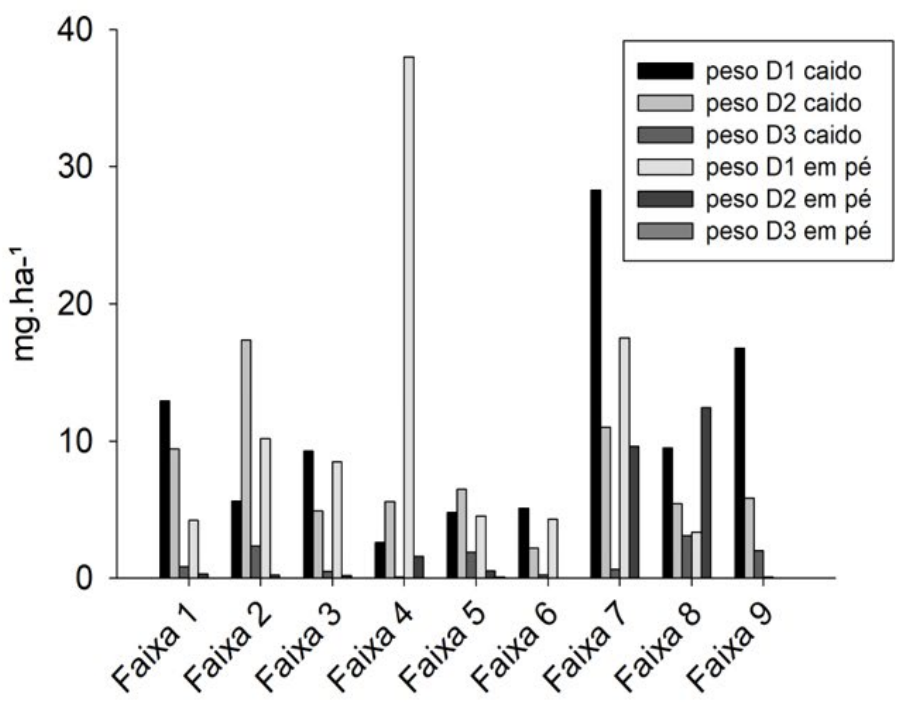

Carbono por grau de decomposição

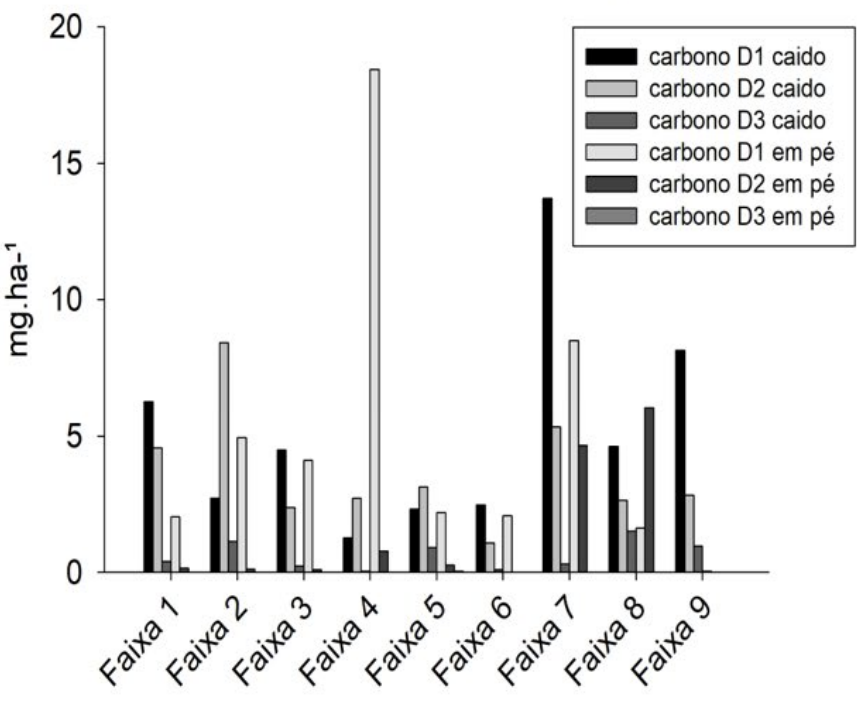

A

B

Figura 3. Peso (A) e quantidade (B) de carbono por faixa, por grau de decomposição e por categoria.

Figure 3. Weight (A) and carbon amount (B) by strip, degree of decomposition and by category.

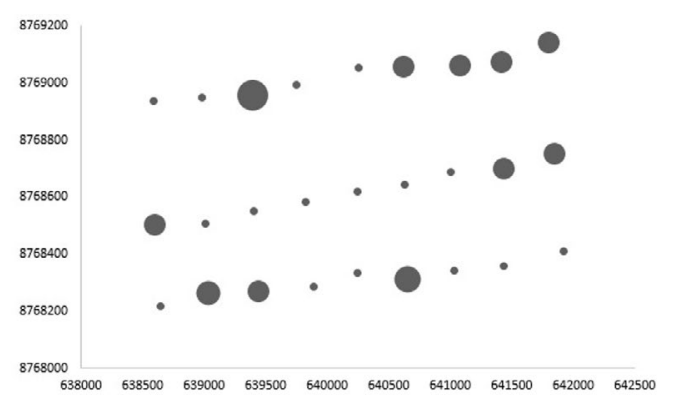

Figura 4. Análise Espacial do volume de necromassa.

Figure 4. Spatial analysis of necromass volume.

\section{Conclusões}

Foi encontrado uma densidade de 732 indivíduos caídos e 78 mortos em pé por hectare. Sendo que a maior concentração de matéria morta se encontra caída representando $54,9 \%$, em contrapartida a matéria morta em pé representa um $45 \%$; sendo os fragmentos registrados de peças com até $8,7 \mathrm{~cm}$ de diâmetro, com decomposição intermediária cerca de 46,5 \% com grua de decomposição intermediário e 27,2 \% com grau de decomposição inicial.

Os resultados mostram que a floresta sob manejo recupera seus estoques de peso, volume e carbono

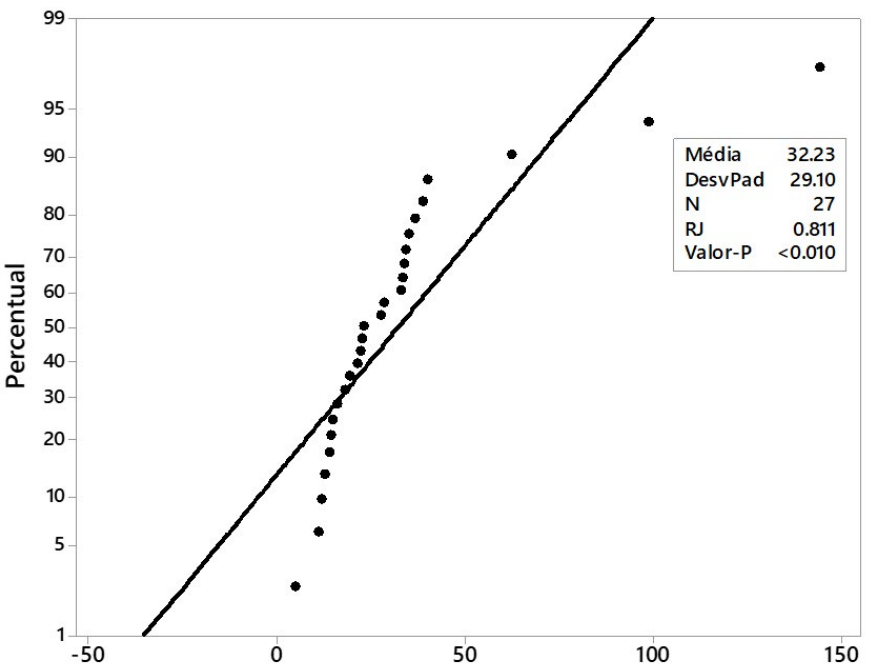

Figura 5. Teste de Normalidade de Shapiro-Wilk.

Figure 5. Shapiro-Wilk Normality Test.

pois os valores encontrados são iguais ou maiores aos valores encontrados em outras áreas semelhantes não manejadas, fazendo com que os valores evidenciem a importância da necromassa no estoque total de carbono pela floresta.

Há ausência de normalidade e ausência de dependência espacial para o conjunto de dados, indicando a 
heterogeneidade da variável necromassa na área de estudo.

A necromassa contribui, mesmo que em menor proporção que a biomassa viva, para a redução da concentração do $\mathrm{CO}_{2}$ na atmosfera e para a diminuição do efeito estufa. Contribuindo para a manutenção, tanto da matéria orgânica quanto do carbono orgânico, auxiliando na recomposição e na ciclagem de nutrientes e evidencia a importância fixação de carbono.

\section{Referências}

[1] Y. Malhi; J. Grace, "Tropical forests and atmospheric carbon dioxide", Trends in ecology and evolution, v 15, no. 8, p 332-337, Setp, 2000

[2] S. Luyssaert; E.D. Schulze; A. Börner, A. Knohl; D. Hessenmöller; B.E. Law; P. Ciais; J. Grace, "Old-growth forests as global carbon sinks" Nature, Vol 455, p. 213-215, Set, 2008.

[3] O. L. Phillips; Y. Malhi; N. Higuchi, "Changes in the Carbon Balance of Tropical Forests: Evidence from Long-term Plots", Science, vol. 282, no. 5388, pp. 439-442, Oct 1998.

[4] H. E. M. Nascimento; W. F. Laurance, "Total Above-ground Biomass in Central Amazonian Rainforests: A landscapescale Study", Forest Ecology and Management, vol. 168, no. 1-3, p. 311-321, Set, 2002.

[5] H.K. Gibbs; S. Brown; J.O. Niles, "Monitoring and estimating tropical Forest carbon stocks: making REDD a reality", Environmental Research Letters, vol. 2, no. 4, pp. 13, Dec, 2007.

[6] S. brown, Estimating biomass and biomass change of tropical forests: A Primer. Forest Resources Assessment Publication, FAO, 1997.

[7] M. Palace; M. Keller; G.P. Asner; J.N.M. Silva; P. Carlos, "Necromass in undisturbed and logged forests in the Brazilian Amazon", Forest Ecology and Management, vol. 238, no.1-3, p. 309-318, Jan, 2007.

[8] E. J, Almeida; B. E. Valério; J. C. Noronha; R. M. Miranda, L. F. Barbosa, M. Machiner; L. A. Pezzini; F. G. Cabeceira; D. C. Lima; D. J. Rodrigues, "Estimativa de necromassa em área de floresta na Amazônia Meridional a partir de duas metodologias distintas", Anais do II Simpósio Cenbam e PPBIO Amazônia Ocidental, Manaus, 2013.
[9] C.W. Woodall; J. Rondeux; P.J. Verkerk; G. Stahl, "Estimating dead wood during national forest inventories: a review of inventory methodologies and suggestions for harmonization", Environmental Management, vol. 44, no. 4, p. 624-631, Aug, 2009.

[10] A. Ribeiro; S. Pellico Neto; D. Stall, "Proposta metodológica para realização de um inventário florestal de necromassa: um estudo de caso", Scientia Forestalis, Piracicaba, vol. 40, no. 93, p. 121-127, Mar, 2012.

[11] D. Pauletto, "Estoque, produção e fluxo de nutrientes da liteira grossa em floresta submetida à exploração seletiva de madeira no noroeste do Mato Grosso", Mestrado em Manejo Florestal, Instituto Nacional de Pesquisas da Amazônia, Manaus, 2006.

[12] P. C. Do Nascimento; J. L. Lani; H. J. O. Zoffoli, "Caracterização, classificação e gênese de solos hidromórficos em regiões litorâneas do Estado do Espírito Santo", Científica, vol. 41, n. 1, p. 82-93, 2013

[13] L. A. G. C. Silva, "Biomas presentes no Estado do Tocantins", Consultoria Legislativa Nota Técnica Câmara dos Deputados, Brasília, DF,Brasil, 2007. p. 2-9.

[14] G. Woldendorp; R. J. Keenan; S. Barry; R. D. Spencer, "Analysis of sampling methods for coarse woody debris", Forest Ecology and Management, vol.198, no. 1-3, p.133148, mar. 2004

[15] M. W. Palace; M. Keller; G. C. Hurtt; S. Frolking, "A review of above ground necromass in tropical forests", in: Tropical Forests, InTech, 2012. p. 215-252.

[16] M. E. Harmon; J. Sexton, "Guidelines for measurements of woody detritus in forest ecosystems". US LTER Network Office, University of Washington, Seatle, WA, USA, n. 20, 1996.

[17] K.L. Waddell, "Sampling coarse woody debris for multiple attributes in extensive resource inventories", Ecological Indicators, vol.1, no.3, p. 139-153, Mar, 2002.

[18] A. J. N. L. Lima, "Avaliação de um sistema de Inventário Florestal Contínuo em áreas manejadas e não manejadas do Estado do Amazonas (AM)", Doutorado em Ciências de Florestas Tropicais, Instituto Nacional de Pesquisas da Amazônia, INPA/UFAM, Manaus, 2010.

[19] D.J. Cardoso; A.C. Vibrans; D.V. Lingner, "Inventário da necromassa florestal caída no chão nos remanescentes 
florestais em Santa Catarina". In: Inventário Florístico Florestal de Santa Catarina, Blumenau, Ed. FURB, p. 217$227,2012$.

[20] M. Keller; M. Palace; G. P. Asner; R. J. Pereira; J. N. M. Silva, "Coarse woody debris in undisturbed and logged forests in the eastern Brazilian Amazon”, Global Change Biology, vol. 10, no.5, p. 784-795, Apr, 2004.

[21] S. A Vieira; L. F. Alves; P. J. Duarte-Neto; S. C Martins; L. G. Veiga; M. A. Scaranello; M. C. Picollo; P. B. Camargo; J. B. Do, Carmo; E. S. Neto; F. A. M. Santos; C. A. Joly; L. A. Martinelli, "Stocks of carbon and nitrogen and partitioning between above- and belowground pools in the Brazilian coastal Atlantic Forest elevation range", Ecology and evolution, vol. 1, no. 3, p. 421-434, Oct, 2011.

[22] G.C.B. Maas, "Compartimentação do estoque de carbono em florestam ombrófila mista", Doutorado em Engenharia Florestal, Universidade Federal do Paraná, Curitiba, 2015.

[23] R. Silvestre, "Comparação da florística, estrutura e padrão espacial em três fragmentos de Floresta Ombrófila Mista no estado do Paraná". Mestrado em Ciências Florestais, Setor de Ciências Agrárias, Universidade Federal do Paraná, Curitiba, PR, 2009.

[24] G. Woldendorp; R.D. Spencer; R.J. Keenan; S. Barry, "An analysis of sampling methods for coarse woody debris in Australian forest ecosystems". Canberra: BRS - Bureau of Rural Science, Report for the National Greenhouse Strategy, Module 6.6. 2002.

[25] R. E. Guimarães, "Inventário De Necromassa Em Remanescentes Florestais No Estado Do Rio De Janeiro", MBA em Gestão Florestal, Departamento de Economia Rural e Extensão, Setor de Ciências Agrárias da Universidade Federal do Paraná 2015.

[26] R.D. Ottmar, R.E. Vihnanek, H.S. Miranda, M.N. Sato, and S.M. Andrade, "Stereo photo series for quantifying Cerrado fuels in Central Brazil - Volume I", [Brochure], Tech. Rep. PNWGTR-519. Portland, OR: U.S. Department of Agriculture, Forest Service, Pacific Northwest Research Station, 2001.

[27] F. S. Lucca, "Estoques de necromassa em um cerrado sensu stricto e uma Floresta Ombrófila Densa Montana, no estado de São Paulo". Tese de Doutorado, Instituto de Botânica da Secretaria de Estado do Meio Ambiente, 2011.

[28] R. M. de Britez; M. Borgo; G. Tiepolo; A. Ferretti; M. Calmon; R. Higa, "Estoque e incremento de carbono em florestas e povoamentos de espécies arbóreas com ênfase na Floresta
Atlântica do Sul do Brasil", Sociedade de Pesquisa em Vida Selvagem e Educacao Ambiental, Embrapa Florestas; Curitiba, 2007.

[29] L.E. Guimarães; I. Roitman; F. Venturoli, "Biomassa Aérea e Fatores de Expansão de uma floresta Estacional Decidual em Niquelândia, Goiás", Rev. Tree dimensional, vol.4, no.7, p. 1, 2019.

[30] C. R. Sanquetta; A. P. D. Corte; C. Pinto; L. A. N. Melo, "Biomass and carbon in non-woody vegetation, dead wood and litter in Iguaçu National Park", Floresta, vol. 44, no. 2, p. 185-194, 2014.

[31] F.C. Freitas; C.P. De Azevedo; C. R. Souza, "Estoque de necromassa em floresta não manejada e floresta manejada na Amazônia Central", Scientia Forestales, vol. 47, no. 121, p. 13-23, mar, 2019.

[32] C. W. Woodall; C. H. Perry; J. A. Westfall, "An empirical assessment of forest floor carbon stock components across the United States", Forest Ecology and Management, vol. 269, p. 1-9, Apr, 2012.

[33] J. Pasher; D. J. King, "Mapping dead wood distribution in a temperate hardwood forest using high-resolution airborne imagery", Forest Ecology and Management, vol. 258, no.7, p. 1536-1548, Set, 2009. 